\title{
On Omega-Languages Defined by Mean-Payoff Conditions
}

\author{
Rajeev Alur ${ }^{1}$, Aldric Degorre $^{2}$, Oded $_{\text {Maler }}{ }^{2}$, and Gera Weiss ${ }^{1}$ \\ 1 Dept. of Computer and Information Science, University of Pennsylvania, USA \\ \{alur,gera\}@cis.upenn.edu \\ 2 CNRS - Verimag, University of Grenoble, France \\ \{aldric.degorre, oded.maler\}@imag. fr
}

\begin{abstract}
In quantitative verification, system states/transitions have associated payoffs, and these are used to associate mean-payoffs with infinite behaviors. In this paper, we propose to define $\omega$-languages via Boolean queries over meanpayoffs. Requirements concerning averages such as "the number of messages lost is negligible" are not $\omega$-regular, but specifiable in our framework. We show that, for closure under intersection, one needs to consider multi-dimensional payoffs. We argue that the acceptance condition needs to examine the set of accumulation points of sequences of mean-payoffs of prefixes, and give a precise characterization of such sets. We propose the class of multi-threshold mean-payoff languages using acceptance conditions that are Boolean combinations of inequalities comparing the minimal or maximal accumulation point along some coordinate with a constant threshold. For this class of languages, we study expressiveness, closure properties, analyzability, and Borel complexity.
\end{abstract}

\section{Introduction}

In algorithmic verification of reactive systems, the system is modeled as a finite-state transition system (possibly with fairness constraints), and requirements are captured as languages of infinite words over system observations [8, 9]. The most commonly used framework for requirements is the class of $\omega$-regular languages. This class is expressive enough to capture many natural requirements, and has well-understood and appealing theoretical properties: it is closed under Boolean operations, it is definable by finite automata (such as deterministic parity automata or nondeterministic Büchi automata), it contains Linear Temporal Logic LTL, and decision problems such as emptiness, language inclusion are decidable [10,11].

The classical verification framework only captures qualitative aspects of system behavior, and in order to describe quantitative aspects, for example, consumption of resources such as CPU and energy, a variety of extensions of system models, logics, and automata have been proposed and studied in recent years [1, 3, 5, 7]. The best known, and the most relevant to our work, approach is as follows: a payoff (or a cost) is associated with each state (or transition) of the model, the mean-payoff of a finite run is simply the average of the payoffs of all the states in the run, and the mean-payoff of an infinite run is the limit, as $n$ goes to infinity, of the mean-payoff of the prefix of length $n$. The notion of mean-payoff objectives was first studied in classical game theory, and 
more recently in verification literature [5, 6, 12]. Most of this work is focused on computing the optimal mean-payoff value, typically in the setting of two-player games, and the fascinating connections between the mean-payoff and parity games.

In this paper, we propose and study ways of defining languages of infinite words based on the mean-payoffs. As a motivating example, suppose 1 denotes the condition that "message is delivered" and 0 denotes the condition that "message is lost." A behavior of the network is an infinite sequence over $\{0,1\}$. Requirements such as "no message is ever lost" (always 1), "only finitely many messages are lost" (eventually-always 1), and "infinitely many messages are delivered" (infinitely-often 1), are all $\omega$-regular languages. However, the natural requirement that "the number of lost messages is negligible" is not $\omega$-regular. Such a requirement can be formally captured if we can refer to averages. For this purpose, we can associate a payoff with each symbol, payoff 0 with message lost and payoff 1 with message delivered, and require that mean-payoff of every infinite behavior is 1 . As this example indicates, using mean-payoffs to define acceptance conditions can express meaningful, non-regular, and yet analyzable, requirements.

The central technical question for this paper is to define a precise query language for mapping mean-payoffs of infinite runs into Boolean answers so that the resulting class of $\omega$-languages has desirable properties concerning closure, expressiveness, and analyzability. The obvious candidate for turning mean-payoffs into acceptance criteria is threshold queries of the form "is mean-payoff above (or below) a given threshold $t$ ". Indeed, this is implicitly the choice in the existing literature on decision problems related to mean-payoff models [5,6,12]. A closer investigation indicates that this is not a satisfactory choice for queries.

First, closure under intersection requires that we should be able to model multiple payoff functions. For this purpose, we define $d$-payoff automata, where $d$ is the dimension of the payoffs, and each edge is annotated with a $d$-dimensional vector of payoffs. We prove that expressiveness strictly increases with the dimension. From the applications point of view, multi-payoffs allow to model requirements involving multiple quantities. Because we allow unbounded dimensions, one can also add coordinates that model weighted sums of the quantities, and put bounds on these coordinates too.

Second, the limit of the mean-payoffs of prefixes of an infinite run may not exist. This leads us to consider the set of accumulation points corresponding to a run. For single-dimensional payoffs, the set of these points is an interval. For multi-dimensional payoffs, we are not aware of existing work on understanding the structure of accumulation points. We establish a precise characterization of the structure of accumulation points: a set can be a set of accumulation points of a run of a payoff automaton if and only if it is closed, bounded, and connected.

Third, if we use $m p$ to refer to the mean-payoff of a run, and consider four types of queries of the form $m p<t, m p \leq t, m p>t$, and $m p \geq t$, where $t$ is a constant, we prove that the resulting four classes of $\omega$-languages have incomparable expressiveness. Consequently acceptance condition needs to support combination of all such queries.

After establishing a number of properties of the accumulation points of multidimensional payoff automata, we propose the class of multi-threshold mean-payofflanguages. For this class, the acceptance condition is a Boolean combination of constraints 
of the form "is there an accumulation point whose $i$ th projection is less than a given threshold $t$ ". We show that the expressive power of this class is incomparable to that of the class of $\omega$-regular languages, that this class is closed under Boolean operations and has decidable emptiness problem. We also study its Borel complexity.

\section{Definitions}

\subsection{Multi-payoff Automata}

Multi-payoff automata are defined as automata with labels, called payoffs, attached to transitions. In this paper, payoffs are vectors in a finite dimensional Euclidean space.

Definition 1 ( $d$-Payoff automaton). A $d$-payoff automaton, with $d \in \mathbb{N}$, is a tuple $\left\langle A, Q, q_{0}, \delta, w\right\rangle$ where $A$ and $Q$ are finite sets, representing the alphabet and states of the automaton, respectively; $q_{0} \in Q$ is an initial state; $\delta \in Q \times A \rightarrow Q$ is a total transition function (also considered as a set of transitions $(q, a, \delta(q, a))$ ) and $w: \delta \rightarrow \mathbb{R}^{d}$ is a function that maps each transition to a d-dimensional vector, called payoff.

Note that we consider only deterministic complete automata.

Definition 2. The following notions are defined for payoff automata:

- A finite run of an automaton is a sequence of transitions of the following type: $\left(q_{1}, a_{1}, q_{2}\right)\left(q_{2}, a_{2}, q_{3}\right) \ldots\left(q_{i}, a_{i}, q_{i+1}\right)$. An infinite run is an infinite sequence of transitions such that any prefix is a finite run.

- We denote by $\lambda(r)$ the word of the symbols labelling the successive transitions of the run $r$, i.e. $\lambda\left(\left(q_{1}, a_{1}, q_{2}\right) \cdots\left(q_{n}, a_{n}, q_{n+1}\right)\right)=a_{1} \cdots a_{n}$.

- A run is initial if $q_{1}=q_{0}$.

- By $\operatorname{run}_{\mathcal{A}}(u)$ we denote the initial run $r$ in $\mathcal{A}$ such that $u=\lambda(r)$

- A cycle is a run $\left(q_{1}, a_{1}, q_{2}\right)\left(q_{2}, a_{2}, q_{3}\right) \ldots\left(q_{i}, a_{i}, q_{i+1}\right)$ such that $q_{1}=q_{i+1}$. A cycle is simple if no proper subsequence is a cycle.

- For a word or run $u, u\lceil n$ denotes the prefix of length $n$ of $u$, and $u[n]$ the nth element of $u$.

- The payoff of a finite run $r$ is payoff $(r)=\sum_{i=1}^{|r|} w(r[i])$.

- The mean-payoff of a run $r$ is $\operatorname{mp}(r)=$ payoff $(r) /|r|$.

- A subset of the states of an automaton is strongly connected if, for any two elements of that subset, there is a path from one to the other.

- A strongly connected component (SCC) is a strongly connected subset that is not contained in any other strongly connected subset.

- A SCC is terminal if it is reachable and there is no path from the SCC to any other SCC.

\subsection{Acceptance}

In the literature, the mean-payoff value of a run is generally associated to the "limit" of the averages of the prefixes of the run. As that limit does not always exist, standard definitions only consider the lim inf of that sequence (or sometimes lim sup) and, 
more specifically, threshold conditions comparing those quantities with fixed constants [2,4,5,12]. As that choice is arbitrary, and more can be said about the properties of that sequence than the properties of just its lim inf or even lim sup, in particular when $d>1$, we choose to consider the entire set of accumulation points of that sequence.

A point $x$ is an accumulation point of the sequence $x_{0}, x_{1}, \ldots$ if, for every open set containing $x$, there are infinitely many indices such that the corresponding elements of the sequence belong to the open set.

Definition 3. We denote by $\operatorname{Acc}\left(x_{n}\right)_{n=1}^{\infty}$ the set of accumulation points of the sequence $\left(x_{n}\right)_{n=1}^{\infty}$. If $r$ is a run of a d-payoff automaton $\mathcal{A}, \operatorname{Acc}_{\mathcal{A}}(r)=\operatorname{Acc}\left(\operatorname{mp}(r\lceil n))_{n=1}^{\infty}\right.$, and for a word $w, \operatorname{Acc}_{\mathcal{A}}(w)=\operatorname{Acc}_{\mathcal{A}}(\operatorname{run}(w))$.

Example 1. Consider the 2-payoff automaton

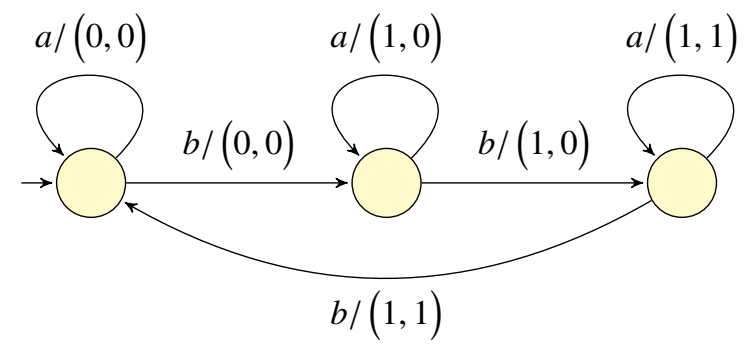

where edges are annotated with expression of the form $\sigma / v$ meaning that the symbol $\sigma$ triggers a transition whose payoff is $v$. Let $w=\prod_{i=0}^{\infty} a^{2^{i}-1} b$ be an infinite word where $b$ 's are isolated by sequences of $a$ 's with exponentially increasing lengths. The $\operatorname{set} \operatorname{Acc}_{\mathcal{A}}(w)$ is the triangle

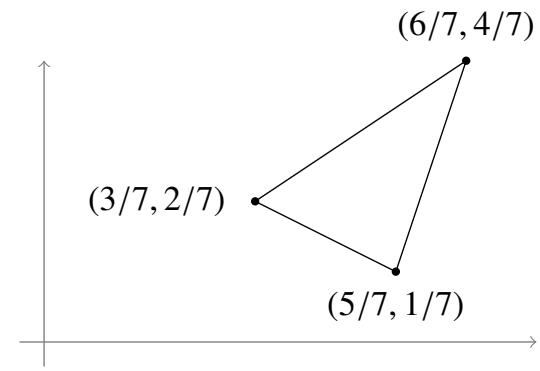

as we show next. By direct calculation we get that $\lim _{n \rightarrow \infty} \operatorname{mp}\left(w \uparrow \sum_{i=0}^{3 n} 2^{i}\right)=(6 / 7,4 / 7)$, $\lim _{n \rightarrow \infty} \operatorname{mp}\left(w \nmid \sum_{i=0}^{3 n+1} 2^{i}\right)=(3 / 7,2 / 7)$, and $\lim _{n \rightarrow \infty} \operatorname{mp}\left(w \uparrow \sum_{i=0}^{3 n+2} 2^{i}\right)=(5 / 7,1 / 7)$. Furthermore, for every $n \in \mathbb{N}, j \in\{0,1,2\}$ and $k \in\left\{0, \ldots, 2^{3 n+j+1}\right\}$, the vector $\operatorname{mp}(w \uparrow$ $\left.k+\sum_{i=0}^{3 n+j} 2^{i}\right)$ is in the convex hull of $\operatorname{mp}\left(w\left\lceil\sum_{i=0}^{3 n+j} 2^{i}\right)\right.$ and $\operatorname{mp}\left(w \uparrow \sum_{i=0}^{3 n+j+1} 2^{i}\right)$ and the maximal distance between points visited on this line goes to zero as $n$ goes to infinity. Together, we get that the points to which the mean-payoff gets arbitrarily close are exactly the points on the boundary of the above triangle. Similarly, if we choose the word $w^{\prime}=\prod_{i=0}^{\infty} a^{3^{i}-1} b$, we get that $\operatorname{Acc}_{\mathcal{A}}\left(w^{\prime}\right)$ is the boundary of the triangle (4/13,3/13), (10/13, 1/13), (12/13,9/13). 
We say that a word or run is convergent, whenever its set of accumulation points is a singleton, i.e. when its sequence of mean payoffs converges. For instance, periodic runs are convergent because the mean-payoffs of the prefixes $r\left\lceil n\right.$ of an infinite run $r=r_{1} r_{2}^{\omega}$ converge to the mean-payoff of the finite run $r_{2}$, when $n$ goes to infinity.

Definition 4. An infinite run $r$ is accepted by a d-payoff automaton $\mathcal{A}$ with condition $F$, where $F$ is a predicate on $2^{\mathbb{R}^{d}}$, if and only if $F\left(\operatorname{Acc}_{\mathcal{A}}(r)\right)$. An infinite word $u$ is accepted if and only if $\operatorname{run}(u)$ is accepted. We denote by $L(\mathcal{A}, F)$ the language of words accepted by $\mathcal{A}$ with condition $F$. In the following, we call mean-payoff language, any language accepted by a d-payoff automaton with such a condition. If $d$ is one and $F(S)$ is of the form $\operatorname{extr} S \bowtie C$ where extr $\in$ \{inf, sup\}, $\bowtie \in\{<, \leq,>, \geq\}$, and $C$ is a real constant; we say that $F$ is a threshold condition.

Example 2. For the 1-payoff automaton

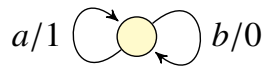

let the acceptance condition $F(S)$ be true iff $S=\{0\}$. This defines the language of words having negligibly many $a$ 's.

\section{Expressiveness}

\subsection{Comparison with $\omega$-Regular Languages}

Before proving specific results on the class of mean-payoff languages, we show that it is incomparable with the class of $\omega$-regular languages. In this context, we call specification types incomparable if each type of specification can express properties that are not expressible in the other type. Incomparability of mean-payoff and $\omega$-regular specifications is, of course, a motivation for studying mean-payoff languages.

We will need the following ad-hoc pumping lemma for $\omega$-regular languages.

Lemma 1 (Pumping lemma). Let $L$ be an $\omega$-regular language. There exists $p \in \mathbb{N}$ such that, for each $w=u_{1} w_{1} u_{2} w_{2} \ldots u_{i} w_{i} \ldots \in L$ such that $\left|w_{i}\right| \geq p$ for all $i$, there are sequences of finite words $\left(x_{i}\right)_{i \in \mathbb{N}},\left(y_{i}\right)_{i \in \mathbb{N}},\left(z_{i}\right)_{i \in \mathbb{N}}$ such that, for all $i, w_{i}=x_{i} y_{i} z_{i},\left|x_{i} y_{i}\right| \leq p$ and $\left|y_{i}\right|>0$ and for every sequence of pumping factors $\left(j_{i}\right)_{i \in \mathbb{N}} \in \mathbb{N}^{\mathbb{N}}$, the pumped word $u_{1} x_{1} y_{1}^{j_{1}} z_{1} u_{2} x_{2} y_{2}^{j_{2}} z_{2} \ldots u_{i} x_{i} y_{i}^{j_{i}} z_{i} \ldots$ is in $L$.

Proof. Similar to the proof of the pumping lemma for finite words.

Proposition 1. There exists a mean-payoff language, defined by a 1-payoff automaton and a threshold acceptance condition, that is not $\omega$-regular.

Proof. Consider the 1-payoff automaton

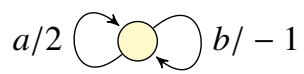

We show that $L=\left\{w \mid \inf \operatorname{mp}_{\mathcal{A}}(w) \leq 0\right\}$ is not regular. For any $p$, the word $w=\left(a^{p} b^{2 p}\right)^{\omega}$ is in that language. Assuming, towards contradiction, that the language is regular and 
using the pumping Lemma 1 on $w$, we can select as factors $w_{i}$ the sequences of $a$ an choose $j_{i}=2$ to obtain a word $w^{\prime}$ that should be in $L$. But since $\mathrm{mp}_{A}\left(w^{\prime}\right)$ is a singleton bigger than zero, $w^{\prime}$ does not satisfy the acceptance condition and therefore is not in $L$, a contradiction.

Proposition 2. There exists an $\omega$-regular language that is not a mean-payoff language.

Proof. Let $L=\left(a^{*} b\right)^{\omega}$. We will show that, in any payoff automaton, we can find two words $u_{1}$ and $u_{2}, u_{1}$ having infinitely often $b$ and $u_{2}$ having eventually only $a$, and such that $\operatorname{Acc}\left(u_{1}\right)=\operatorname{Acc}\left(u_{2}\right)$. Then obviously no general mean-payoff acceptance condition can distinguish those two words although $u_{1} \in L$ and $u_{2} \notin L$.

Let us construct the counter-example. Suppose $\mathcal{A}$ is a payoff automaton recognizing $L$ with some predicate $F$. Let $c_{a}$ be a cycle such that $\lambda\left(c_{a}\right)$ contains only $a$ 's and $c_{b}$ a cycle such that $\lambda\left(c_{b}\right)$ contains at least one $b$, both starting in some state $q$ in a terminal strongly connected component of $\mathcal{A}$, and let $p$ be an initial run leading to $q$.

The mean-payoffs of the run $r=p \prod_{i=1}^{\infty} c_{a}^{i} c_{b}$, which should be accepted, converge to $\operatorname{mp}_{\mathcal{A}}\left(c_{a}\right)$, which is also the mean-payoff of $p c_{a}^{\omega}$, which should be rejected but has to be accepted by $\mathcal{A}$, since it has the same mean-payoff as $r$.

\subsection{Topology of Mean-Payoff Accumulation Points}

In this section we discuss the structure of the set of accumulation points. In particular we characterize the sets that are the accumulation points of some run of a payoff automaton.

If $S$ is a strongly connected component of an automaton, and $C$ is the set of simple cycles in $S$, then we denote by ConvHull( $S)$ the convex hull of $\{\operatorname{mp}(c) \mid c \in C\}$.

Theorem 1. Let $r$ be an infinite run of a d-payoff automaton, then $\operatorname{Acc}(r)$ is a closed, connected and bounded subset of $\mathbb{R}^{d}$.

\section{Proof.}

Closed: True for any set of accumulation points: let $\left(a_{n}\right)$ be a sequence in a topological space, and $\left(x_{n}\right) \in \operatorname{Acc}\left(a_{n}\right)_{n=1}^{\infty}$ be a sequence of accumulation points converging to a point $x$. For any $x_{i}$, we can choose a sub-sequence $\left(a_{i_{n}}\right)$ converging to $x_{i}$. Now we can construct a sub-sequence of elements that converges to $x$ : for every $i$, take the first element $a_{i_{f(i)}}$ of $a_{i_{n}}$ which is at a distance smaller than $2^{-i}$ from $x_{i}$ such that $f(i)>f(i-1)$. Then the sequence $\left(a_{i_{f(i)}}\right)_{i \in \mathbb{N}}$ converges to $x$.

Bounded: As we are speaking of a sequence of averages of the (finite) set of payoffs, it is clear that the sequence of mean-payoffs remains in the convex hull of that set, which is bounded.

Connected: Proof by contradiction. Suppose there exists two disjoint open sets $O_{1}$ and $O_{2}$ such that $A c c(r) \subseteq O_{1} \cup O_{2}$. Let $d$ be the distance between $O_{1}$ and $O_{2}$. As those sets are open and disjoint, $d>0$. But the vector between two successive averages is payoff $(r\lceil n) / n-\operatorname{payoff}(r\lceil n-1) / n-1=(1 / n)(\operatorname{payoff}(r\lceil n-1))+w(r[n])-n /(n-$ 1) $\operatorname{payoff}(r\lceil n-1))=(1 / n)(w(r[n])-\operatorname{mp}(r\lceil n))$, whose norm is smaller than $\Delta / n$, where $\Delta=\max \left\{\left\|w(t)-w\left(t^{\prime}\right)\right\| t, t^{\prime} \in \delta\right\}$. If a run has accumulations points in both $O_{1}$ and $O_{2}$, then there exist $n>\Delta / d$ such that the $n$th step is in $O_{1}$ and the $(n+1)$ th in $O_{2}$. The 
distance between those two points has to be both greater than $d$ and smaller than $\Delta / n$, which is not possible.

As a remark, we can say more than boundedness: indeed a run eventually comes into a SCC it never leaves. The contribution of the payoffs of the SCC becomes then dominant as $n$ goes to the infinity. Even better, actually, the contribution of the simple cycles of that SCC is dominant. Thus the set of accumulation points is included in the convex hull of the simple cycles of the SCC.

The following theorem is a converse to Theorem 1

Theorem 2. For every non-empty, closed, bounded and connected set $D \subset \mathbb{R}^{d}$, there is a d-payoff automaton and a run $r$ of that automaton such that $\operatorname{Acc}(r)=D$.

Proof. Take any automaton with a reachable SCC such that $D$ is contained in the convex hull of the cycles of the SCC.

For every integer $n>0$, let $\left\{O_{i, n}: i=1, \ldots, l_{n}\right\}$ be a finite coverage of $D$ by open sets of diameter smaller than $1 / n$. Such a coverage exists, for example, by covering $D$ by spheres of diameter $1 / n$.

Suppose $p$ is a finite initial run going into the SCC. For every $n$ and every $i$, we can prolong $p$ with a suffix $c$ such that $\operatorname{mp}(p c) \in O_{n, i}$ and $p c$ is in the SCC (form the end of $p$ onwards). For that, we need $c$ to be long enough and have the right proportions of simple cycles. Furthermore, as $\operatorname{mp}(p c \uparrow l+1)-\operatorname{mp}(p c \uparrow l)$ becomes smaller when $l$ goes to infinity, we can make the distance of $\operatorname{mp}(p c \uparrow l)$ from $D$ converge to zero when $l$ goes to infinity.

As the set $\left(O_{n, i}\right)_{n, i \in \mathbb{N} \times \mathbb{N}}$ is countable, we can construct recursively the successive suffixes $c_{1,1}, c_{1,2}, \ldots, c_{2,1}, c_{2,2}, \ldots$ such that $\operatorname{mp}\left(p c_{1,1} c_{1,2} \ldots c_{2,1} c_{2,2} \ldots c_{n, i}\right)$ is in $O_{n, i}$, and such that for every $l, \operatorname{mp}\left(p \prod_{j i \in \mathbb{N} \times \mathbb{N}} c_{j i} \uparrow l\right)$ is at a distance smaller than $K / l$ from $D$.

Let $x \in D$. Then for every $n, x \in O_{n, i}$ for some $i$, thus for every $n$, the sequence of mean-payoffs comes within a radius $1 / n$ from $x$, which means $x$ is an accumulation point. Conversely, if $y \notin D$, as $D$ is closed, it is at a distance $\delta>0$ from $D$, moreover there exist a $l$ such that $\operatorname{mp}(p c \uparrow l)$ never exits a radius $\epsilon<\delta$ around $D$ and therefore the sequence of mean-payoff will never come in a radius $\delta-\epsilon$ from $y$. So $y$ is not an accumulation point. We conclude that $\operatorname{Acc}_{\mathcal{A}}(r)$ is exactly $D$.

Actually, like for Theorem 1, a careful examination of the proof reveals that a stronger statement is true. Specifically, it is easy to verify that any closed, bounded and connected set contained in any of the SCC of an automaton is the set of accumulation points of some run of that automaton.

\subsection{Comparison of Threshold Mean-Payoff Languages}

We study mean-payoff languages where the minimum and maximum of the set of accumulation points are compared with a given threshold. We assume, without loss of generality, that the threshold is zero because changing the threshold is equivalent to an affine transformation of the payoffs. We show that the different threshold languages are incomparable in expressive power. 
Definition 5. We denote by $\mathcal{L}_{\bowtie}$ the class of mean-payoff languages accepted by a 1payoff automaton with the condition $\min \operatorname{Acc}(w) \bowtie 0$, where $\bowtie$ is $<,>, \leq$ or $\geq$.

Note that these languages are the winning conditions used to define mean-payoff games, e.g. in [12], because $\min \operatorname{Acc}(w)=\liminf _{n \rightarrow \infty} \operatorname{mp}_{\mathcal{A}}(w\lceil n)$. We do not need to discuss the class of languages defined as complements of these conditions because $\mathcal{L}_{>}$is co $\mathcal{L}_{\leq}$ and $\mathcal{L}_{\geq}$is co $\mathcal{L}_{<}$, where co $\mathcal{L}_{\bowtie}$ is the set of languages defined as sets of words that do not satisfy $\min \operatorname{Acc}(w) \bowtie 0$ for some automaton.

Theorem 3. The classes $\mathcal{L}_{<}, \mathcal{L}_{\leq}, \mathcal{L}_{\geq}$and $\mathcal{L}_{>}$are incomparable.

Proof. We begin by showing that $\mathcal{L}_{<}$and $\mathcal{L}_{\leq}$are incomparable. Consider the automaton

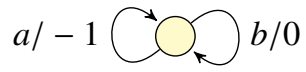

and the language $L=\{w \mid \min \operatorname{Acc}(w)<0\}$. Suppose, towards contradiction, that there exists an automaton $\mathcal{A}^{\prime}$ accepting $L$ with a $\mathcal{L}_{\leq}$condition. Consider $c_{a}$ and $c_{b}$ two cycles in $\mathcal{A}^{\prime}$, labelled respectively with a word in $a(a+b)^{i}$ and with $b^{j}$ for some integers $i$ and $j$, and start from a same reachable state $q$ (two such cycles exist at least in any terminal strongly connected component). Let $p$ be a finite initial run ending at $q$. As $p c_{a}^{\omega}$ is a run of $\mathcal{A}^{\prime}$ which should be accepted, it is necessary that payoff ${ }_{\mathcal{A}^{\prime}}\left(c_{a}\right) \leq 0$, and as $p c_{b}^{\omega}$ should not be accepted, it is necessary that payoff $\mathcal{A}_{\mathcal{A}^{\prime}}\left(c_{b}\right)>0$. For all $k$, the run $p\left(c_{a} c_{b}^{k}\right)^{\omega}$ should be accepted. So it is necessary that for all $k$, payoff ${ }_{\mathcal{A}^{\prime}}\left(c_{a} c_{b}^{k}\right) \leq 0$. Thus payoff $_{\mathcal{A}^{\prime}}\left(c_{a}\right)+k$ payoff $_{\mathcal{A}^{\prime}}\left(c_{b}\right) \leq 0$, which is possible if and only if payoff $\mathcal{A}_{\mathcal{A}^{\prime}}\left(c_{b}\right) \leq 0$ and contradicts payoff $\mathcal{A}^{\prime}\left(c_{b}\right)>0$. Thus $L$ cannot be accepted by a $\mathcal{L}_{\leq}$condition.

Conversely, consider the automaton

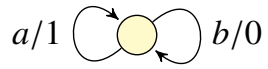

with the language $L$ defined by the $\mathcal{L}_{\leq}$acceptance condition. Towards contradiction, assume that $\mathcal{A}^{\prime}$ is an automaton accepting $L$ with the $\mathcal{L}_{<}$acceptance contradiction. If $c_{a}, c_{b}$ and $p$ are defined in $\mathcal{A}^{\prime}$, the same way as before, we should have payoff $\mathcal{A}_{\mathcal{A}^{\prime}}\left(c_{a}\right) \geq 0$ and payoff ${ }_{\mathcal{A}^{\prime}}\left(c_{b}\right)<0$. For all $k$, the run $p\left(c_{a} c_{b}^{k}\right)$ should be rejected, so it is necessary that payoff $_{\mathcal{A}^{\prime}}\left(c_{a} c_{b}^{k}\right) \geq 0$. Thus for all $k$, payoff ${ }_{\mathcal{A}^{\prime}}\left(c_{a}\right)+k$ payoff $_{\mathcal{A}^{\prime}}\left(c_{b}\right) \geq 0$, which is possible if and only if payoff $\mathcal{A}^{\prime}\left(c_{b}\right) \geq 0$ and contradicts payoff $\mathcal{A}^{\prime}\left(c_{b}\right)<0$. Therefore $L$ cannot be expressed by a $\mathcal{L}_{<}$condition.

These counter examples can be adapted for proving that any class with strict inequality symbol is incomparable to any class with a weak inequality symbol. It remains to prove the incompatibility of $\mathcal{L}_{<}$and $\mathcal{L}_{>}$and that of $\mathcal{L}_{\leq}$and $\mathcal{L}_{\geq}$.

Consider the language $L$ defined by the automaton

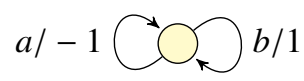

(denoted by $\mathcal{A}$ ) with the $\mathcal{L}_{\leq}$acceptance condition. Suppose, towards contradiction, that $\mathcal{A}^{\prime}$ is an automaton defining the same language with a $\mathcal{L}_{\geq}$condition. Choose two cycles $c_{a}$ and $c_{b}$ starting from two states $q_{a}$ and $q_{b}$ in a same terminal strongly connected component of $\mathcal{A}^{\prime}$, such that $c_{a}$ is labelled by $a^{l}$ and $c_{b}$ is labelled by $b^{m}$ for some $l$ and $m$, an initial run $p$ going to $q_{b}$ and two runs $u_{a b}$ and $u_{b a}$ going from $q_{a}$ to $q_{b}$ and from $q_{b}$ to $q_{a}$, respectively. Because $p u_{b a} c_{a}^{\omega}$ should be accepted and $p c_{b}^{\omega}$ 


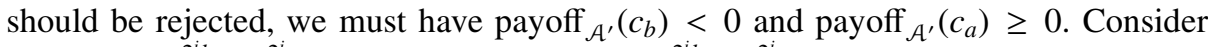
$r=p \prod_{i \in \mathbb{N}} c_{b}^{2^{i} l} u_{b a} c_{a}^{2^{i} m} u_{a b}$. Then $\operatorname{mp}_{\mathcal{A}}\left(\lambda\left(p \prod_{i=0}^{n} c_{b}^{c^{i} l} u_{b a} c_{a}^{2^{i} m} u_{a b}\right)\right)$ converges to 0 as $n$ goes to infinity, thus $\lambda(r) \in L$, and therefore $r$ should be accepted by $\mathcal{A}^{\prime}$ with the $\mathcal{L}_{\geq}$condition. But $\mathrm{mp}_{\mathcal{A}_{3}^{\prime}}\left(\operatorname{word}\left(p \prod_{i=0}^{n} c_{b}^{2^{i} l} u_{b a} c_{a}^{2^{i} m} u_{a b} c_{b}^{2 n+1} l\right)\right)$ converges to a negative limit, thus $r$ cannot be accepted by $\mathcal{A}_{3}^{\prime}$ with the $\mathcal{L}_{\geq}$condition, leading to contradiction.

Conversely, consider the language $L$, defined with the same automaton, but with the $\mathcal{L}_{\geq}$condition. Suppose $\mathcal{A}^{\prime}$ is an automaton defining the same $L$ with the $\mathcal{L}_{\leq}$condition. We can choose two cycles $c_{a}$ and $c_{b}$ starting from two states $q_{a}$ and $q_{b}$ in a same terminal strongly connected component of $\mathcal{A}^{\prime}$ such that $c_{a}$ is labelled by $a^{l}$ and $c_{b}$ is labelled by $b^{m}$ for some $l$ and $m$, an initial run $p$ going to $q_{b}$ and two runs $u_{a b}$ and $u_{b a}$ going from $q_{a}$ to $q_{b}$ and from $q_{b}$ to $q_{a}$. Then we should have payoff $\mathcal{A}_{\mathcal{A}^{\prime}}\left(c_{b}\right) \leq 0$ and payoff $\mathcal{A}_{\mathcal{A}^{\prime}}\left(c_{a}\right)>0$ (because $p u_{b a} c_{a}^{\omega}$ should be rejected and $p c_{b}^{\omega}$ should be accepted). Consider $r=p \prod_{i \in \mathbb{N}} u_{b a} c_{a}^{2^{i} m} u_{a b} c_{b}^{2^{i} l}$. Then $\operatorname{mp}_{\mathcal{A}_{3}}\left(\lambda\left(p\left(\prod_{i=0}^{n} u_{b a} c_{a}^{i^{i} m} u_{a b} c_{b}^{2^{i} l}\right) u_{b a} c_{a}^{2^{n+1} m}\right)\right)$ converges to a negative limit as $n$ goes to infinity, thus $\lambda(r) \notin L$, and therefore $r$ should be rejected by $\mathcal{A}^{\prime}$ with the $\mathcal{L}_{\leq}$condition. But $\mathrm{mp}_{\mathcal{A}^{\prime}}\left(\lambda\left(p \prod_{i=0}^{n} u_{b a} c_{a}^{2^{i} m} u_{a b} c_{b}^{i^{i}}\right)\right)$ converges to 0 , thus $r$ is accepted by $\mathcal{A}^{\prime}$ with the $\mathcal{L}_{\leq}$condition, which contradicts the fact that it recognizes $L$.

We have thus established the incomparability of $\mathcal{L}_{\geq}$and $\mathcal{L}_{\leq}$. As $\mathcal{L}_{<}$and $\mathcal{L}_{>}$are the classes of the complements of languages in respectively $\mathcal{L}_{\geq}$and $\mathcal{L}_{\leq}$, it also implies the incomparability of the latter pair.

The incompatibility of threshold classes shows how arbitrary the choice of only one of them as a standard definition would be. This suggests definition of a larger class including all of them.

\subsection{Mean-Payoff Languages in the Borel Hierarchy}

For a topological space $X$, we denote by $\Sigma_{1}^{0}$ the set of open subsets by and $\Pi_{1}^{0}$ the set of closed subsets. The Borel hierarchy is defined inductively as the two sequences $\left(\Sigma_{\alpha}^{0}\right)$ and $\left(\Pi_{\alpha}^{0}\right)$, where $\Sigma_{\alpha}^{0}=\left(\bigcup_{\beta<\alpha} \Pi_{\beta}^{0}\right)_{\sigma}$, and $\Pi_{\alpha}^{0}=\left(\bigcup_{\beta<\alpha} \Sigma_{\beta}^{0}\right)_{\delta}$, where $\alpha$ and $\beta$ are ordinals and $(\bullet)_{\sigma},(\bullet)_{\delta}$ denote closures under countable intersections and unions, respectively.

We consider the standard topology over $A^{\omega}$ with the base $\left\{w A^{\omega}: w \in A^{*}\right\}$, i.e. a subset of $A^{\omega}$ is open if and only if it is a union of sets, each set consists of all possible continuations of a finite word.

Theorem 4. The following facts hold: $\mathcal{L}_{\leq} \subset \Pi_{2}^{0}, \mathcal{L}_{\leq} \nsubseteq \Sigma_{2}^{0}, \mathcal{L}_{<} \subset \Sigma_{3}^{0}$ and $\mathcal{L}_{<} \nsubseteq \Pi_{3}^{0}$.

Proof. - Let $L \in \mathcal{L}_{\leq}$, then there exists $d \in \mathbb{N}$, a 1-payoff automaton $\mathcal{A}$ such that $L=\left\{w \in A^{\omega} \mid \min \left(\operatorname{Acc}_{\mathcal{A}}(w)\right) \leq 0\right\}$. Therefore we can write $L$ as

$$
\begin{aligned}
L & =\bigcap_{N \in \mathbb{N}}\left\{w \in A^{\omega} \mid \forall m \in \mathbb{N} \exists n>m \mathrm{mp}_{\mathcal{A}}(w\lceil n)<1 / N)\right\} \\
& =\bigcap_{N \in \mathbb{N}, m \in \mathbb{N}} \bigcup_{n>m}\left\{w \in A^{\omega} \mid \mathrm{mp}_{\mathcal{A}}(w\lceil n)<1 / N)\right\} .
\end{aligned}
$$

For any $N$ and $m$ the condition $\operatorname{mp}_{\mathcal{A}}(w\lceil n)<1 / N)$ is independent of the suffix past the $n$th symbol of $w$ and therefore the set $\left\{w \in A^{\omega} \mid \mathrm{mp}_{\mathcal{A}}(w\lceil n)<1 / N)\right\}$ is clopen. We get that $\mathcal{L}_{\leq} \in \Pi_{2}^{0}$. 
- We prove $\mathcal{L}_{>} \nsubseteq \Pi_{2}^{0}$, which is the same as $\mathcal{L}_{\leq} \nsubseteq \Sigma_{2}^{0}$ because $\mathcal{L}_{>}=$co $\mathcal{L}_{\leq}$. Let $L$ be the set of words on alphabet $A=\{a, b\}$ having more than negligibly many $b$. We already demonstrated that $L \in \mathcal{L}_{>}$. Suppose $L \in \Pi_{2}^{0}$. Then $L=\bigcap_{i \in \mathbb{N}} L_{i} A^{\omega}$ for some family of languages of finite words $L_{i}$. We can assume without loss of generality that the words of $L_{i}$ have all length $i$. For all $m$, the word $w_{m}=\left(\prod_{j=1}^{m-1} a^{2^{j}} b\right)\left(a^{2^{m}} b\right)^{\omega} \in L$ (as it is ultimately periodic with a period where the proportion of $b$ is not 0 ). For the word $w=\prod_{j=1}^{\infty} a^{2^{j}} b$, it means that any prefix $w \uparrow i$ of length $i$ is in $L_{i}$. This is a contradiction, because $w \notin L$.

- For the two last items of the theorem: Chatterjee exhibited in [2] a $\Pi_{3}^{0}$-hard language in $\mathcal{L}_{\geq}$. He also established that this class is included in $\Pi_{3}^{0}$. As $\mathcal{L}_{\geq}=\operatorname{co} \mathcal{L}_{<}$, that proves what we need.

\subsection{Dimensionality}

In this section we analyze closure properties of mean-payoff languages defined by automata with a fixed dimension.

The following lemma shows that, for any $d$, the class of mean-payoff languages definable by $d$-payoff automata is not closed under intersection.

Lemma 2. If $d_{1}$ and $d_{2}$ are two integers, then there exists $L_{1}$ and $L_{2}$, two mean-payoff languages of dimensions $d_{1}$ and $d_{2}$ such that $L_{1}$ and $L_{2}$ contain only convergent words and $L_{1} \cap L_{2}$ is not definable as a dimension d mean-payoff language with $d<d_{1}+d_{2}$.

Proof. Let $A=\left\{a_{1}, \ldots, a_{d_{1}}\right\}$ and $B=\left\{b_{1}, \ldots, b_{d_{2}}\right\}$ be two disjoint alphabets. Let $\mathcal{A}_{1}$ be the one-state $d_{1}$-payoff automaton on alphabet $A \cup B$, such that the payoff of the transition $\left(q_{0}, a_{i}, q_{0}\right)$ is 1 on the $i$ th coordinate and 0 in the other coordinates and the payoff of the transition $\left(q_{0}, b_{i}, q_{0}\right)$ is 0 . And let $\mathcal{A}_{2}$ be the $d_{2}$-payoff one-state automaton defined similarly by swapping $a$ and $b$.

Let $L_{i}$ be the language defined on $\mathcal{A}_{i}$ by predicate $F_{i}$, testing equality with the singleton $\left\{l_{i}\right\}$, where $l_{i}$ is in the simplex defined by the $d_{i}+1$ different payoffs of the transitions of $\mathcal{A}_{i}$. In the proof of Theorem 2 we establish that the $L_{i}$ are not empty.

Let $w \in(A+B)^{\omega}$, then $w$ is in $L_{1}$ if and only if the proportion of $a_{i}$ in every prefix tends to the $i$ th coordinate of $l_{1}$, and it is in $L_{2}$ if and only if the proportion of $b_{i}$ in every prefix tends to the $i$ th coordinate of $l_{2}$.

Then for $w$ to be in $L_{1} \cap L_{2}$, it is necessary that the proportion of every symbols tends to either a coordinate of $l_{1}$, if that symbol is a $a_{i}$, or a coordinate of $l_{2}$, if it is a $b_{i}$.

Now suppose $L_{1} \cap L_{2}$ is recognized by a $d$-payoff automaton with $d<d_{1}+d_{2}$. Choose one terminal strongly connected component of $\mathcal{A}$ and consider for every symbol $a$ of the alphabet a cycle labeled by a word in $a^{*}$, starting at some state $q_{a}$. Let also be $p$ an initial run going to $q_{a}$ and for every pair of symbols $a, b$ a path $u_{a b}$ going from $q_{a}$ to $q_{b}$.

Only looking at the runs in the language $p\left\{u_{a_{1} a} c_{a}^{*} u_{a a_{1}} \mid a \in A \cup B\right\}^{\omega}$, it is possible to converge to any proportion of the symbols of $A \cup B$, and thus have runs whose labeling word is in $L$. But as the payoffs are in dimension $d$, and the number of symbols is $d_{1}+d_{2}>d$, that language also contains runs converging to different symbol proportions but still having the same mean-payoff limit. Those runs are accepted by $\mathcal{A}$ but are not labeled by a word in $L$. 
Next, we prove that the intersection of two languages of dimensions $d_{1}$ and $d_{2}$ is a language of dimension $d_{1}+d_{2}$. This will be proved constructively, by showing that the intersection language is the language defined on the product automaton with the "product" condition. Before going to the statement of the lemma, we need to define what those products are.

Definition 6. If $F_{1}$ and $F_{2}$ are predicates on $2^{\mathbb{R}^{d_{1}}}$ and $2^{\mathbb{R}^{d_{2}}}$, we denote by $F_{1} \cap F_{2}$ the predicate on $2^{\mathbb{R}^{d_{1}+d_{2}}}$ which is true for $X \subseteq \mathbb{R}^{d_{1}+d_{2}}$ if and only if $F_{1}\left(p_{1}(X)\right)$ and $F_{2}\left(p_{2}(X)\right)$, where $p_{1}$ is the projection on the $d_{1}$ first coordinates and $p_{2}$ on the $d_{2}$ last.

Definition 7 (Weighted automata product). If $\mathcal{A}_{1}=\left\langle A, Q_{1}, q_{0}^{1}, \delta_{1}, w_{1}\right\rangle$ is a d d-payoff automaton and $\mathcal{A}_{2}=\left\langle A, Q_{2}, q_{0}^{2}, \delta_{2}, w_{2}\right\rangle$, a d $d_{2}$-payoff automaton, then we define $\mathcal{A}_{1} \otimes$ $\mathcal{A}_{2}=\left\langle A, Q_{1} \times Q_{2},\left(q_{0}^{1}, q_{0}^{2}\right), \delta_{1 \otimes 2}, w_{1 \otimes 2}\right\rangle$, the product of $\mathcal{A}_{1}$ and $\mathcal{A}_{2}$, a $\left(d_{1}+d_{2}\right)$-payoff automaton such that

- $\delta_{1 \otimes 2}=\left\{\left(\left(q_{1}, q_{2}\right), a,\left(q_{1}^{\prime}, q_{2}^{\prime}\right)\right) \mid\left(q_{1}, a, q_{1}^{\prime}\right) \in \delta_{1} \wedge\left(q_{2}, a, q_{2}^{\prime}\right) \in \delta_{2} \wedge a \in A\right\}$,

- $w_{1 \otimes 2}: \delta_{1 \otimes 2} \rightarrow \mathbb{R}^{d_{1}+d_{2}}$ is such that if $w_{1}\left(q_{1}, a, q_{1}^{\prime}\right)=\left(x_{1}, \ldots x_{d_{1}}\right)$ and $w_{2}\left(q_{2}, a, q_{2}^{\prime}\right)=$ $\left(y_{1}, \ldots y_{d_{2}}\right)$, then $w\left(\left(q_{1}, q_{2}\right), a,\left(q_{1}^{\prime}, q_{2}^{\prime}\right)\right)=\left(x_{1}, \ldots x_{d_{1}}, y_{1}, \ldots y_{d_{2}}\right)$.

But before we state the theorem, we need the following lemma:

Lemma 3. If $r$ is a run of a d-payoff automaton $\mathcal{A}$ and $p$ is a projection from $\mathbb{R}^{d}$ to $\mathbb{R}^{d^{\prime}}$, with $d^{\prime}<d$, then $\operatorname{Acc}\left(p\left(\operatorname{mp}_{\mathcal{A}}(r\lceil n))\right)=p\left(\operatorname{Acc}\left(\operatorname{mp}_{\mathcal{A}}(r\lceil n))\right)\right.\right.$

Proof. Let $x^{\prime} \in \operatorname{Acc}\left(p\left(\operatorname{mp}_{\mathcal{A}}(r\lceil n))\right)\right.$. For any $i \in \mathbb{N}, p\left(\operatorname{mp}_{\mathcal{A}}(r\lceil n))\right.$ eventually comes into a distance $1 / i$ from $x^{\prime}$, for some index $n_{i}$. For $j>i \operatorname{mp}_{\mathcal{A}}\left(r \uparrow n_{j}\right)$ remains in $B\left(x^{\prime}, 1 / i\right) \times K$ (where $K$ is a compact of $\mathbb{R}^{d-d^{\prime}}$ ), as this product is compact, it has at least one accumulation point. Thus the distance from $x^{\prime}$ to $p\left(\operatorname{Acc}\left(\operatorname{mp}_{\mathcal{A}}(r\lceil n))\right)\right.$ is $0 . \operatorname{But} \operatorname{Acc}\left(\operatorname{mp}_{\mathcal{A}}(r\lceil n))\right.$ is a closed set and $p$, being a projection, is continuous, so $p(\operatorname{Acc}(\operatorname{mp}(r\lceil n)))$ is closed too, which means $x^{\prime} \in p\left(\operatorname{Acc}\left(\operatorname{mp}_{\mathcal{A}}(r\lceil n))\right)\right.$, and $\operatorname{so} \operatorname{Acc}\left(p(\operatorname{mp}(r\lceil n))) \subseteq p\left(\operatorname{Acc}_{\left(m_{\mathcal{A}}\right.}(r\lceil n))\right)\right.$.

Conversely, if $x^{\prime} \in p\left(\operatorname{Acc}\left(\operatorname{mp}_{\mathcal{A}}(r\lceil n))\right)\right.$ a sub-sequence $\operatorname{mp}_{\mathcal{A}}\left(r\left\lceil n_{i}\right)\right.$ converges to a $x$ such that $x^{\prime}=p(x)$, and thus $p\left(\mathrm{mp}_{\mathcal{A}}\left(r \uparrow n_{i}\right)\right)$ converges to $x^{\prime}$, which means $x^{\prime} \in$ $\operatorname{Acc}\left(p(\operatorname{mp}(r\lceil n)))\right.$. We conclude that $\operatorname{Acc}\left(p\left(\operatorname{mp}_{\mathcal{A}}(r\lceil n))\right)=p\left(\operatorname{Acc}\left(\operatorname{mp}_{\mathcal{A}}(r\lceil n))\right)\right.\right.$.

Now we have all the needed tools, we can characterize the intersection of two meanpayoff languages as another mean-payoff language defined on an automaton whose dimension is known.

Proposition 3. For any two $d_{1}$-payoff and $d_{2}$-payoff automata $\mathcal{A}_{1}$ and $\mathcal{A}_{2}$ and any two predicates $F_{1}$ and $F_{2}$ on respectively $2^{\mathbb{R}^{d_{1}}}$ and $2^{\mathbb{R}^{d_{2}}}$, the following equality holds: $L\left(\mathcal{A}_{1}, F_{1}\right) \cap L\left(\mathcal{A}_{2}, F_{2}\right)=L\left(\mathcal{A}_{1} \otimes \mathcal{A}_{2}, F_{1} \cap F_{2}\right)$.

Proof. Suppose $u \in A^{\omega}$, then the sequence of mean-payoffs of run $r$ of $u$ in $\mathcal{A}_{1} \otimes \mathcal{A}_{2}$ are the projections by $p_{1}$ and $p_{2}$ of the sequence of mean-payoffs of some runs $r_{1}$ and $r_{2}$ in $\mathcal{A}_{1}$ and $r_{2}$ in $\mathcal{A}_{2}$ whose labeling is $u$. And conversely, if $u$ has runs $r_{1}$ and $r_{2}$ in $\mathcal{A}_{1}$ and $r_{2}$ in $\mathcal{A}_{2}$, then it has a run $r$ in $\mathcal{A}_{1} \otimes \mathcal{A}_{2}$ whose sequence of mean-payoffs projects by $p_{1}$ and $p_{2}$ onto those of $r_{1}$ and $r_{2}$.

If $r, r_{1}$, and $r_{2}$ are such runs (the payoffs of $r$ projecting on those of $r_{1}$ and $r_{2}$ ), then using lemma 3, we find that $\operatorname{Acc}_{\mathcal{A}}\left(r_{1}\right)=\operatorname{Acc}\left(p_{1}(\operatorname{mp}(r\lceil n)))=p_{1}(\operatorname{Acc}(\operatorname{mp}(r\lceil n)))\right.$ and that $\operatorname{Acc}_{\mathcal{A}}\left(r_{2}\right)=\operatorname{Acc}\left(p_{2}(\operatorname{mp}(r\lceil n)))=p_{2}(\operatorname{Acc}(\operatorname{mp}(r\lceil n)))\right.$. 
But by definition $\left(F_{1} \cap F_{2}\right)(\operatorname{Acc}(\operatorname{mp}(r \uparrow n)))$ holds iff $F_{1}\left(p_{1}(\operatorname{Acc}(\operatorname{mp}(r \uparrow n)))\right)$ and $F_{2}\left(p_{2}(\operatorname{Acc}(\operatorname{mp}(r\lceil n))))\right.$ hold, thus it holds iff $F_{1}\left(\operatorname{Acc}_{\mathcal{A}_{1}}\left(r_{1}\right)\right)$ and $F_{2}\left(\operatorname{Acc}_{\mathcal{A}_{2}}\left(r_{2}\right)\right)$ hold.

From that we deduce that a word is in $L\left(\mathcal{A} \otimes \mathcal{A}, F_{1} \cap F_{2}\right)$ if and only if it is both in $L\left(\mathcal{A}_{1}, F_{1}\right)$ and $L\left(\mathcal{A}_{2}, F_{2}\right)$.

\section{An Analyzable Class of Mean-Payoff Languages}

\subsection{The Class of Multi-threshold Mean-Payoff Languages}

As a candidate for a class of mean-payoff languages that is closed under complementation and includes all the expected standard mean-payoff language classes, we propose the following definition.

Definition 8. A language $L$ is a multi-threshold mean-payofflanguage (denoted by $L \in$ $\left.\mathcal{L}_{m t}\right)$ if it is the mean-payoff language defined on some d-payoff automaton $\mathcal{A}$, with a predicate $F$ such that $F(S)$ is a Boolean combination of threshold conditions on $p_{i}(S)$ where $p_{i}$ is the projection along the ith coordinate.

Example 3. Consider the automaton given in Example 1 and the multi-threshold meanpayoff language $L=\left\{w \mid \min p_{1}(\operatorname{Acc}(w))>.1 \wedge \max p_{1}(\operatorname{Acc}(w))<.9 \wedge \min p_{2}(\operatorname{Acc}(w))\right.$ $\left.>.1 \wedge \max p_{2}(\operatorname{Acc}(w))<.9\right\}$. For the word $w$, defined in Example 1, the set of accumulation points is shown to be a triangle that is contained in the box $\left\{x \mid .1<p_{1}(x)<\right.$ $\left..9 \wedge .1<p_{2}(x)<.9\right\}$ and therefore $w \in L$.

Geometrically, multi-threshold acceptance conditions can be visualized as specifying constraints on the maximal and minimal projection of $\operatorname{Acc}(w)$ on the axes. Since we can extend the payoff vectors by adding a coordinate whose values are a linear combination of the other coordinates, also threshold constraints involving minimal and maximal elements of the projection of $\operatorname{Acc}(w)$ on other lines are expressible, as shown in the following example.

Example 4. Assume that, with the automaton given in Example 1, we want to accept the words $w$ such that $\operatorname{Acc}(w)$ is contained in the triangle $(.2, .2)-(.8, .2)-(.2, .8)$. We can do so by extending the dimension of the payoffs and renaming $(0,0) \mapsto(0,0,0),(1,0) \mapsto$ $(1,0,1)$, and $(1,1) \mapsto(1,1,2)$. Namely, by adding a coordinate whose value is the sum of the other two coordinates. Then, $L=\left\{w \mid \min p_{1}(\operatorname{Acc}(w))>.2 \wedge \min p_{2}(\operatorname{Acc}(w))>\right.$ $\left..2 \wedge \max p_{3}(\operatorname{Acc}(w))<1\right\}$ is the wanted language.

\subsection{Closure under Boolean Operations}

We prove here that $\mathcal{L}_{m t}$ is in fact the Boolean closure of $\mathcal{L}_{\S} \triangleq \mathcal{L}_{<} \cup \mathcal{L}_{\leq} \cup \mathcal{L}_{>} \cup \mathcal{L}_{\geq}$.

Theorem 5. $\mathcal{L}_{m t}$ is closed under Boolean operations and any language in $\mathcal{L}_{m t}$ is a Boolean combination of languages in $\mathcal{L}_{\lesseqgtr}$.

Proof. Closure by complementation: let $L$ be a $\mathcal{L}_{m t}$ language, defined on some automaton $\mathcal{A}$ by a predicate $P . w \in L$ iff $P\left(\operatorname{Acc}_{\mathcal{A}}(w)\right)$. So $w \in L^{c}$ iff $w \notin L$, that is iff 
$\neg P\left(\operatorname{Acc}_{\mathcal{A}}(w)\right)$. But $\neg P$ is also a Boolean combination of threshold conditions, thus $L^{c}$ is a $\mathcal{L}_{m t}$ language.

Closure by intersection: let $L_{1}$ and $L_{2}$ be two $\mathcal{L}_{m t}$ languages defined respectively on the automata $\mathcal{A}$ and $\mathcal{A}$ by the predicates $P_{1}$ and $P_{2}$. Then $L_{1} \cap L_{2}=L\left(\mathcal{A} \otimes \mathcal{A}, P_{1} \cap P_{2}\right)$ (Theorem 3). It is easy to see that $P_{1} \cap P_{2}$ is still a Boolean combination of thresholds, and thus $L\left(\mathcal{A} \otimes \mathcal{A}, P_{1} \cap P_{2}\right)$ is in $\mathcal{L}_{m t}$.

The other Boolean operations can be written as a combination of complementation and intersection.

Now we show, by induction on height of the formula of a predicate, that any $\mathcal{L}_{m t}$ language is a Boolean combination of $\mathcal{L}_{\lessgtr}$ languages.

We can, without loss of generality, suppose that every threshold concerns a different coordinate (if a coordinate has several thresholds, we can duplicate that coordinate, keeping the same values, and the language will remain the same). We can also assume that the predicate is written only with combinations of conjunctions and negations of thresholds.

- If the height is 0 , that means that the condition is only one threshold on a multipayoff automaton. The recognized language is the same as that of the automaton projected on the tested coordinate, so it is in $\mathcal{L}_{\S}$.

- If the predicate is $\neg P$, then the recognized language is the complement of $L(\mathcal{A}, P)$, which is a Boolean combination of $\mathcal{L}_{m t}$ languages of lesser height.

- If the predicate is $P=P_{1} \wedge P_{2}$, let us call $\mathcal{A}_{i}$, a copy of $\mathcal{A}$ whose payoffs are projected on the subspace tested in $P_{i}$. Then $\mathcal{A}$ is isomorphic to $\mathcal{A}_{1} \otimes \mathcal{A}_{2}$. Furthermore, as the set of coordinates that are tested in $P_{1}$ and $P_{2}$ are disjoint, their exists some $P_{1}^{\prime}$ and $P_{2}^{\prime}$ with the same heights as $P_{1}$ and $P_{2}$, such that $P=P_{1}^{\prime} \cap P_{2}^{\prime}$. Thus $L=L\left(\mathcal{A}_{1}, P_{1}^{\prime}\right) \cap L\left(\mathcal{A}_{2}, P_{2}^{\prime}\right)$ (Theorem 3 , which is a Boolean combination of $\mathcal{L}_{m t}$ languages of lesser height.

\subsection{Decidability}

Theorem 6. The emptiness of a language of $\mathcal{L}_{m t}$ is decidable.

Proof. We can assume the predicate of acceptance is written in disjunctive normal form (if not, we can find an equivalent DNF formula). Then we can see that a run is accepted whenever its set of accumulation points satisfies at least one of the disjuncts, and in a disjunct, every literal has to be satisfied. If we know how to check if a literal is satisfied, then this provides an obvious decision algorithm for one run.

Then it is easy to see that there are two types of literal. Some say that there must exist an accumulation point whose tested coordinate is greater or smaller than the threshold, we call those existential literals. The other literals say that every accumulation point should have the tested coordinate above or below the threshold, those we call universal literals.

For checking the emptiness of $L(\mathcal{A}, F)$, we propose the following algorithm: Try, for every a disjunct of $F$ and every reachable SCC $C$ of $\mathcal{A}$, to compute $P(C)$, the convex hull of the payoffs of its transitions, then compute $C^{\prime}$ the intersection of $P(C)$ with every universal literal, and finally check whether it intersects with every existential literal of 
the disjunct. If it does, then return true, else loop. If you exhausted the combinations, return false.

If this algorithm returns true, because $C^{\prime}$ is a convex polyhedron included in $C$ and intersecting with every existential literal, we can construct $D$ which is connected, closed, included in $C^{\prime}$, and intersects with every existential literal (take for instance the convex hull of a family consisting in one point in every intersection of $C^{\prime}$ with an existential literal). We can see that $F(D)$ holds. Then, Theorem 2 says there exist a run $r$ such that $\operatorname{Acc}_{\mathcal{A}}(r)=D$, and thus there exist a word which that run and therefore is in $L(\mathcal{A}, F)$.

If that algorithm returns false, for every reachable SCC $C$, if you choose a closed connected subset $D$ of $P(C)$ (as Theorem 1 says sets of accumulation points have to be), then for every disjunct, $D$ either is not completely included in some universal literal, either does not intersect with some existential literal. In both case, $D$ does not make the disjunct true. So $F$ holds for no set of accumulation points of a run of $\mathcal{A}$, which implies that $L(\mathcal{A}, F)$ is empty.

\section{Summary and Future Directions}

We proposed a definition of $\omega$-languages using Boolean combination of threshold predicates over mean-payoffs. This type of specifications allows to express requirements concerning averages such as "no more than $10 \%$ of the messages are lost" or "the number of messages lost is negligible". The later is not expressible by $\omega$-regular requirements. We showed that if closure under intersection is needed, multi-dimensional payoffs have to be considered. For runs of $d$-payoff automata, we studied acceptance conditions that examine the set of accumulation points and characterized those sets as all closed, bounded and connected subsets of $\mathbb{R}^{d}$.

The class of multi-threshold mean-payoff languages was proposed, using acceptance conditions that are Boolean combinations of inequalities comparing the minimal or maximal accumulation point along some coordinate with a constant threshold. We studied expressiveness, closure properties, analyzability, and Borel complexity.

Possible direction for future include extension to non-deterministic automata, and the study of multi-mean-payoff games.

\section{Acknowledgments}

This research was partially supported by NSF grants CNS 0524059 and CPA 0541149 , and the French Minalogic project Athole.

\section{References}

1. Alur, R., Kanade, A., Weiss, G.: Ranking automata and games for prioritized requirements. In: Gupta, A., Malik, S. (eds.) CAV 2008. LNCS, vol. 5123, pp. 240-253. Springer, Heidelberg (2008)

2. Chatterjee, K.: Concurrent games with tail objectives. Theor. Comput. Sci. 388(1-3), 181198 (2007) 
3. Chatterjee, K., de Alfaro, L., Henzinger, T.: The complexity of quantitative concurrent parity games. In: Proceedings of the 17th ACM-SIAM Symposium on Discrete Algorithms, pp. 678-687 (2006)

4. Chatterjee, K., Doyen, L., Henzinger, T.A.: Quantitative languages. In: Kaminski, M., Martini, S. (eds.) CSL 2008. LNCS, vol. 5213, pp. 385-400. Springer, Heidelberg (2008)

5. Chatterjee, K., Henzinger, T., Jurdziński, M.: Mean-payoff parity games. In: Proceedings of the 20th Annual Symposium on Logic in Computer Science, pp. 178-187. IEEE Computer Society Press, Los Alamitos (2005)

6. Gimbert, H., Zielonka, W.: Deterministic priority mean-payoff games as limits of discounted games. In: Bugliesi, M., Preneel, B., Sassone, V., Wegener, I. (eds.) ICALP 2006. LNCS, vol. 4052, pp. 312-323. Springer, Heidelberg (2006)

7. Kupferman, O., Lustig, Y.: Lattice automata. In: Cook, B., Podelski, A. (eds.) VMCAI 2007. LNCS, vol. 4349, pp. 199-213. Springer, Heidelberg (2007)

8. Manna, Z., Pnueli, A.: The temporal logic of reactive and concurrent systems: Specification. Springer, Heidelberg (1991)

9. Pnueli, A.: The temporal logic of programs. In: 18th IEEE Symposium on the Foundations of Computer Science (FOCS 1977), pp. 46-57 (1977)

10. Thomas, W.: Automata on infinite objects. In: van Leeuwen, J. (ed.) Handbook of Theoretical Computer Science, vol. B, pp. 133-191. Elsevier, Amsterdam (1990)

11. Vardi, M., Wolper, P.: Reasoning about infinite computations. Information and Computation 115(1), 1-37 (1994)

12. Zwick, U., Paterson, M.: The complexity of mean payoff games on graphs. Theoretical Computer Science 158, 343-359 (1996) 